(2) Open Access Full Text Article

\title{
In vivo gastric residence and gastroprotective effect of floating gastroretentive tablet of DA-960I, an extract of Artemisia asiatica, in beagle dogs
}

This article was published in the following Dove Press journal:

Drug Design, Development and Therapy

9 June 2016

Number of times this article has been viewed

\author{
Jeong Soo Kim' \\ Kwang Ho Cha' \\ Seung Yeob Kang' \\ Donghan Won' \\ Sun Woo Jang' \\ Miwon Son' \\ Moon Ho Son' \\ Ho Jung $\mathrm{Choi}^{2}$ \\ Young Won Lee ${ }^{2}$ \\ Myung Joo Kang ${ }^{3}$ \\ 'Dong-A Pharmaceutical Co. Ltd., \\ Giheung-gu, Yongin, Gyeonggi, ${ }^{2}$ College \\ of Veterinary Medicine and Research \\ Institute of Veterinary Medicine, \\ Chungnam National University, \\ Daejeon, ${ }^{3}$ College of Pharmacy, \\ Dankook University, Dongnam-gu, \\ Cheonan, Chungnam, South Korea
}

Correspondence: Myung Joo Kang College of Pharmacy, Dankook University, I 9 Dandae-ro, Dongnam-gu, Cheonan, Chungnam 330-7I4, South Korea

Tel +82 4l 550 I446

Fax +82415507899

Email kangmj@dankook.ac.kr
Objective: DA-9601, an extract of Artemisia asiatica containing eupatilin and jaceosidin as active compounds, has been prescribed to treat gastritis in Asia. In recent times, sustainedrelease, floating gastroretentive (GR) tablets of DA-9601 are available on the market. In the present study, the physical properties and in vitro drug release profile, in vivo gastric residence time, and gastroprotective effect of GR tablet were compared to those of immediate release (IR) tablets of DA-9601.

Method: In vitro buoyancy behavior (floating lag time and duration) and release profile of eupatilin were assessed in acidic medium. The in vivo intragastric behaviors of the barium sulfate-loaded IR and GR tablets were evaluated in beagle dogs by radiographic studies. Local gastroprotective effect was compared in an experimentally induced gastric lesion in beagle dogs after oral administration of IR (three times per day) or GR (twice daily) tablets for 15 days.

Results: Upon contact with gastric juice, a low-density floating tablet (apparent density of $0.93 \mathrm{~g} / \mathrm{cm}^{3}$ ) was buoyant on the medium and was upheld for 14 hours, providing sustained drug release profile, whereas the IR tablet disintegrated within 10 minutes, showing complete drug release within 2 hours. In vivo radiographic studies showed that the GR tablet was retained for $>4$ hours in the stomach. Both DA-9601 formulations remarkably alleviated gastric mucosal injury compared to placebo group, when observed by gastric endoscopy.

Conclusion: Twice-daily GR tablets exhibited a prolonged gastric residence time and a remarkable mucosal restoration effect in animal models. Therefore, the GR system of DA-9601 could be a substitute dosage form for the treatment of gastritis, while reducing the dosing frequency and thus improving patient compliance.

Keywords: DA-9601, gastroretentive tablet, controlled release, radiographic studies, gastroprotective effects

\section{Introduction}

The standardized ethanol extract of Artemisia asiatica, named DA-9601, has been widely prescribed for the treatment of patients with gastric mucosal lesions of acute and chronic gastritis in Asia. DA-9601 contains the pharmacologically active compounds eupatilin and jaceosidin, which have been demonstrated to possess potent anti-inflammatory and/or protective activities in gastrointestinal lesions, in both animal models and even humans. ${ }^{1,2}$ Currently, the immediate release (IR) dosage form of DA-9601 (Stillen ${ }^{\mathrm{TM}}$; Dong-A Pharmaceutical Co. Ltd., Seoul, South Korea) is administered three times a day. However, this dosing regimen impairs patient compliance, which ultimately reduces overall efficacy. Thus, the necessity for frequent daily dosing with an IR formulation has led to the need for a prolonged release dosage form of DA-9601. 
Gastroretentive (GR) oral dosage forms have been exploited to provide sustained release (SR) to better patient compliance by reducing the dosing frequency, improve the oral absorption of drugs with a narrow absorption window, or enhance the local pharmacological effects of drugs in the gastric region, by increasing the residence time in the stomach..$^{3-5}$ Several formulation approaches, such as expendable, mucoadhesive, sedimentation, and floating systems, have been employed to extend the gastric retention time. ${ }^{6,7}$ Among them, an effervescent floating system consisting of a gas-forming agent in combination with the swellable polymers is one of the established approaches to prepare the GR dosage form. ${ }^{8}$ Carbonates and/or bicarbonates in the matrix react with hydrogen ion in the gastric fluid and generate carbon dioxide bubbles. The bubbles uphold the swollen matrix to float over the gastric contents, without affecting the rate of gastric emptying and/or peristaltic waves. ${ }^{3,9}$ However, conventional effervescent system requires a lag time for buoyancy and intragastric behavior is significantly affected by gastric $\mathrm{pH}$ and food intake. Alternatively, low-density GR systems possessing intrinsic density lower than that of gastric fluids have been devised to provide rapid onset of floating in the stomach, complementing the drawback of conventional gas-generating system..$^{10,11} \mathrm{Oh}$ et $\mathrm{al}^{11}$ reported that low-density GR tablets (apparent density of $0.98 \mathrm{~g} / \mathrm{cm}^{3}$ ) of metformin prepared by sublimation method floated for over 24 hours with no floating lag time.

Recently, we formulated twice-daily, floating GR tablet of DA-9601 (Stillen 2X) to achieve prolonged drug release in the gastric region and to maximize its local gastroprotective effect. ${ }^{12}$ To build a low-density GR system of DA-9601, we combined the effervescent floating system with low-density porous materials to float on the gastric juice with no lag time. Then, the bicarbonate in the matrix was converted to carbon dioxide in the presence of gastric acid, and the bubbles trapped in the swollen matrix kept the dosage form afloat in stomach. However, to develop and recommend the novel SR tablet for use in clinical applications, supplemental information on gastric residence time, dissolution profile, and pharmacological effects is essentially needed. In the present study, in vitro buoyance behavior and dissolution profile of the GR tablet of DA-9601 (Stillen 2X) were compared with those of IR tablet. In vivo gastric residence time of the GR tablet was assessed in beagle dogs using radiographic study. Moreover, the local gastroprotective effect of the GR system was evaluated in a gastric mucosal lesion-induced beagle dog model by gastric endoscopy and compared with IR formulations.

\section{Materials and methods Materials}

Standardized ethanol extract of A. asiatica and commercialized IR tablet dosage forms of DA-9601 were provided by Dong-A Pharmaceutical Co. Ltd. Eupatilin (purity over $99 \% \mathrm{w} / \mathrm{w}$ ) hydrochloride and sodium lauryl sulfate were purchased from Sigma-Aldrich Co. (St Louis, MO, USA). Copovidone (Kollidon VA64) and poloxamer P407 were kindly provided by BASF Co. Ltd. (Ludwigshafen, Germany). Hydroxypropyl methylcellulose (HPMC K4M) was obtained from Shin-Etsu Chemical Co. (Tokyo, Japan). Microcrystalline cellulose (Avicel ${ }^{\circledR}$ PH 101) was obtained from Asahi Kasei (Tokyo, Japan). All other chemicals used were of reagent grade and were used without further purification.

\section{Animals}

Female beagle dogs (Central Lab. Animal Inc., Gyeonggi-do, South Korea) weighing 7-12 kg were used to evaluate in vivo gastric residence time and in vivo pharmacological effect of the DA-9601 GR system. Animals were kept under standard laboratory conditions and given access to a standard diet and water ad libitum. The animal study was approved by the Institutional Animal Care and Use Committee of Dong-A Pharmaceutical Co. Ltd. in Seoul, and was carried out in accordance with the National Institutes of Health guideline "Principles of Laboratory Animal Care" (National Institutes of Health publication no 85-23, revised 1996).

\section{Preparation of GR tablets}

The floating tablet of DA-9601 was prepared by wet granulation and tabletization, followed by the film coating process. Standardized ethanol extract of A. asiatica, copovidone, and poloxamer P407 were dissolved in $70 \%(\mathrm{v} / \mathrm{v})$ ethanolic solution. The binding solution was added drop-wise to the mixture of microcrystalline cellulose, calcium silicate, sodium bicarbonate, crospovidone, and HPMC and granulated in the fluid bed granulator (UniGlatt; Glatt ${ }^{\circledR} \mathrm{GmbH}$, Dresden, Germany) at $40^{\circ} \mathrm{C}$. The dried granules were lubricated with magnesium stearate, and then compressed into tablets using a single-punch tablet pressing machine (Erweka, Heusenstamm, Germany) equipped with $14.5 \times 8.2 \mathrm{~mm}^{2}$ oval-shaped punch. The compressed tablets were coated with polyvinyl alcohol-based moisture-proof coating agent (Opadry ${ }^{\circledR}$ amb; Colorcon Inc., Harleysville, PA, USA) using a pan coater under the following conditions: rotating speed $10 \mathrm{rpm}$, spraying speed $5 \mathrm{~mL} / \mathrm{min}$, and inlet air temperature $70^{\circ} \mathrm{C}$. 


\section{Physical characterization of IR and GR tablets}

The diameter and thickness of the compressed tablets were measured using digital caliper. The compressed DA-9601 tablets were tested for crushing strength using a hardness tester (TBH $310 \mathrm{MD}$, Erweka). A tablet was placed between two plates and then upper plate was moved down to break the tablet. The breaking force required to break or damage the solid dosage form was recorded. The friability of the IR and GR tablets was evaluated using a TA 10 Rochetype friability tester (Erweka). Tablets were placed in the friabilator which was then operated at a speed of $25 \mathrm{rpm}$ for 4 minutes. Five measurements were performed for each formulation $(n=5)$.

\section{Drug content}

Each tablet ( $\mathrm{n}=5)$ was transferred to a $200 \mathrm{~mL}$ volumetric flask containing $70 \%(\mathrm{v} / \mathrm{v})$ ethanolic solution and sonicated for 60 minutes. The samples were then centrifuged at $12,000 \mathrm{rpm}$ for 10 minutes to remove insoluble excipients. Then, the concentration of eupatilin, one of the active ingredients of DA-9601, in the supernatants was analyzed by high-performance liquid chromatography (HPLC). Chromatographic analysis for eupatilin was carried out using an L2200 HPLC system (Hitachi Ltd., Tokyo, Japan), which consisted of an L2130 pump, an L2400 UV-vis (Ultravioletvisible) detector, and an Ezchrom Elite integrator (version 3.1.3). Samples $(10 \mu \mathrm{L})$ were applied to an Inertsil ODS-II column ( $4.6 \times 150 \mathrm{~mm}, 5 \mu \mathrm{m}$; GL Science, Tokyo, Japan) and isocratically eluted using a mobile phase consisting of $1 \%$ ammonium acetate buffer ( $0.5 \%$ acetic acid) and acetonitrile $(58: 42, \mathrm{v} / \mathrm{v})$ at a flow rate $1 \mathrm{~mL} / \mathrm{min}$. UV absorbance was monitored at $350 \mathrm{~nm}$.

\section{In vitro buoyancy behavior}

The in vitro buoyancy of the tablets was determined by measuring the floating lag times and duration of buoyancy according to the method described by Rosa et al. ${ }^{13}$ Each tablet $(\mathrm{n}=5)$ was placed in a $150 \mathrm{~mL}$ beaker containing $100 \mathrm{~mL}$ of $0.1 \mathrm{~N}$ hydrochloride. The time required for the tablet to rise to the surface and float was taken as the floating lag time. The time for which the tablets kept floating was termed as floating duration.

\section{Swelling behavior of GR tablets}

The swelling property of the GR tablets was determined by measuring the $\%$ weight gain of swollen tablet. The GR tablets were immersed in $200 \mathrm{~mL}$ of $0.1 \mathrm{~N}$ hydrochloride at $37^{\circ} \mathrm{C}$ and no rotation was provided. After $0.5,1,2,4,6,8$, and 12 hours, the immersed tablets were removed from the medium and immediately blotted with paper towel to remove surface droplets. The percentage of swelling at each time point was estimated using the following equation:

$$
\% \text { swelling }=\frac{W_{2}-W_{1}}{W_{1}}
$$

where $W_{1}$ is the initial weight of the tablet and $W_{2}$ is the weight of the swollen tablets. The experiment was performed in sixplicate.

\section{In vitro dissolution test and release kinetics}

Dissolution profiles of eupatilin from the tablets were assessed according to the USP XXVIII paddle method (Varian Industries, Palo Alto, CA, USA). Each tablet $(\mathrm{n}=6)$ was put into $900 \mathrm{~mL}$ of simulated gastric juice (pH 1.2) containing $0.2 \%$ sodium lauryl sulfate without any other sinking device, so that the tablet would float on the surface of the medium. The stirring speed was $50 \mathrm{rpm}$, and the temperature was maintained at $37^{\circ} \mathrm{C} \pm 0.1^{\circ} \mathrm{C}$. Approximately $4 \mathrm{~mL}$ aliquots were withdrawn at various time intervals and filtered using a $0.45 \mu \mathrm{m}$ glass membrane syringe filter. At each sampling time, an equal volume of the test medium was replaced. Filtered samples were appropriately diluted with methanol, and the drug concentration was assayed by HPLC.

Release kinetics of eupatilin, a pharmacologically active compound of DA-9601, from the GR tablet were mathematically investigated by fitting the release data from 0 to 8 hours to the following equations: zero-order equation ${ }^{14}$ : $Q_{\mathrm{t}}=k_{0} \mathrm{t}$, where $Q_{\mathrm{t}}$ is the percentage of drug released at time $\mathrm{t}$ and $k_{0}$ is the release rate constant; first-order equation ${ }^{15}$ : In $\left(100-Q_{\mathrm{t}}\right)=\operatorname{in} 100-k_{1} \mathrm{t}$, where $k_{1}$ is the release rate constant; Higuchi's equation ${ }^{16}: Q_{\mathrm{t}}=k_{\mathrm{H}} \mathrm{t}^{1 / 2}$, where $k_{\mathrm{H}}$ is the Higuchi release rate constant; Korsmeyer-Peppas equation ${ }^{17}$ : $Q_{\mathrm{t}} / Q_{\infty}=k_{\mathrm{KP}} \mathrm{t}^{n}$, where $Q_{\mathrm{t}} / Q_{\infty}$ is the portion of drug released at time $\mathrm{t}, k_{\mathrm{KP}}$ is a release rate constant, and $n$ is the release exponent.

\section{In vivo radiographic studies}

The in vivo gastric residence time of the IR and GR tablets of DA-9601 was evaluated in beagle dogs by radiographic studies as previously reported. ${ }^{18}$ For the radiographic study, IR and GR tablets containing $80 \mathrm{mg}$ of barium sulfate as a radio opaque agent were additionally prepared by the originator. Beagle dogs ( $\mathrm{n}=3$ per group) were fasted overnight 
and were only allowed free access to water. Approximately 30 minutes before drug dosing, the beagle dogs received standardized chow (Greenbia TF; Dr Chung's Food Co., Ltd., Seoul, South Korea). Then, one tablet was administered orally to each dog with $250 \mathrm{~mL}$ of water. Radiographs were obtained using X-ray equipment after time intervals of immediately after oral administration and at $0.5,1,2,4$, and 6 hours after oral administration. During the period of study, beagles were only allowed free access to water.

\section{In vivo gastroprotective effect in gastritis- induced beagle dogs}

The local gastroprotective effect of GR tablet of DA-9601 in comparison to the placebo and IR formulation was evaluated using a total of 18 female beagle dogs weighing $7-12 \mathrm{~kg}$ with gastric ulcers as previously reported, ${ }^{19,20}$ with slight modifications. Beagle dogs were assigned to three groups ( $n=6$ per group) by a stratified randomization scheme designed to achieve similar group mean body weights. The dogs were anesthetized by intravenous injection of alfaxalone $(0.02 \mathrm{mg} / \mathrm{kg})$ and isoflurane inhalation. Stomach walls were confirmed to be normal by gastric endoscopy. Mucosal lesions were then induced by making an injury of uniform size, $3 \mathrm{~mm}$ in diameter and $3 \mathrm{~mm}$ in depth, using biopsy forceps.

Placebo tablet or IR tablet containing $60 \mathrm{mg}$ of DA-9601 was orally administered to the dogs three times per day after meals for 15 days. The GR tablet containing $90 \mathrm{mg}$ of DA-9601 was administered two times per day for 15 days. Gastric endoscopy was performed on the mornings of inspection days, 1-2 hours after drug administration. Restoration effects were evaluated using a $10 \mathrm{~mm}$ gastric endoscope (Fujinon LMP-SD) and were estimated by grading mucosal lesions from 0 to 5 , where " 0 " indicated a normal stomach wall, "1" a scar with similar to normal gastric wall, "2" insignificant erosion with flare, "3" severe erosion with flare and swelling, "4" a gastric ulcer with a blood spot, and grade " 5 " a bleeding gastric lesion.

\section{Statistical analysis}

All the statistical testing was performed using KruskalWallis test analysis. A $P$-value of $<0.05$ was considered to indicate significant differences, unless otherwise indicated.

\section{Results}

\section{Physical properties of DA-960I IR and GR tablets}

The mechanical and/or physicochemical properties of the IR and GR tablets of DA-9601, such as dimensions, hardness, friability, apparent density, and drug content, are summarized in Table 1. The dimension and thickness of GR tablet containing larger quantity of DA-9601 (75 mg) were $\sim 0.9$ and $0.6 \mathrm{~mm}$ larger than those of IR tablet (50 $\mathrm{mg}$ ), respectively. The hardness of the both products was between 12.2 and $17.7 \mathrm{kp}$ and the friability was below $0.1 \%$, showing satisfactory physical characteristics. The drug content in both compositions was uniform with low values of standard deviation. However, the apparent density was quite different between the two formulations. The apparent density of GR tablet was $0.93 \mathrm{~g} / \mathrm{cm}^{3}$, while that of IR tablet was $1.35 \mathrm{~g} / \mathrm{cm}^{3}$.

\section{In vitro buoyancy, swelling behavior, and dissolution profile}

IR and GR tablets of DA-9601 were assessed in terms of floating lag time and duration, swelling behavior, and drug release profiles in acidic medium. In case of IR tablet, it could not maintain matrix integrity, but was rapidly eroded within 10 minutes (Figure 1). On the other hand, GR tablets were rapidly drifted onto gastric juices upon contact with the gastric fluid, due to its low density $\left(0.93 \mathrm{~g} / \mathrm{cm}^{3}\right)$. The swollen tablet maintained the matrix integrity and exhibited a total buoyance time of 14 hours in aqueous medium. The percentage of water uptake of the GR tablets in acidic medium as a function of time is plotted in Figure 2. The swelling ratio of GR tablet containing crospovidone and HPMC gradually increased as time elapsed and reached a plateau at 8 hours with the maximum percentage of water uptake being $113 \%$. The value slightly lowered at 12 hours to $91 \%$.

The IR formulation showed an initial burst release ( $\sim 80 \%$ in 30 minutes) as well as a faster release pattern, achieving complete drug release after 2 hours (Figure 3). On the other hand, the drug release from the GR tablet was

Table I Physicochemical and in vitro floating properties of the IR and GR tablets of DA-960I

\begin{tabular}{|c|c|c|}
\hline Characteristics & IR tablet & GR tablet \\
\hline \multicolumn{3}{|l|}{ Physicochemical characteristics } \\
\hline Diameter $(\mathrm{mm})^{\mathrm{a}}$ & $13.4 \times 7.6 \times 4.4$ & $|4.5 \times 8.2 \times 5|$. \\
\hline Total weight $(\mathrm{mg})^{\mathrm{b}}$ & $340.2 \pm 2.7$ & $411.3 \pm 3.3$ \\
\hline Hardness $(\mathrm{kp})^{\mathrm{b}}$ & $17.7 \pm 0.5$ & $12.2 \pm 0.5$ \\
\hline Friability (\%) & $0.15 \pm 0.01$ & $0.13 \pm 0.01$ \\
\hline Apparent density $\left(\mathrm{g} / \mathrm{cm}^{3}\right)^{\mathrm{b}}$ & $1.35 \pm 0.05$ & $0.93 \pm 0.02$ \\
\hline Eupatilin content (mg) & $0.95 \pm 0.01$ & $1.43 \pm 0.01$ \\
\hline \multicolumn{3}{|l|}{ In vitro floating capacity } \\
\hline Lag time (minutes) & Sink & Immediately \\
\hline Floating duration (hours) ${ }^{\mathrm{b}}$ & Sink & $14.0 \pm 0.5$ \\
\hline
\end{tabular}

Notes: axpressed as major axis $\times$ minor axis $\times$ thickness. ${ }^{\text {bData }}$ are expressed as mean $\pm S D$ values $(n=5)$. Apparent density $\left(g / \mathrm{cm}^{3}\right)$ was calculated by the Rhinoceros ${ }^{\mathrm{TM}}$ program (McNeel North America, Seattle, WA, USA) after estimating the dimensions of the tablet.

Abbreviations: DA-960I, a standardized ethanol extract of Artemisia asiatica; GR, gastroretentive; IR, immediate release; SD, standard deviation. 


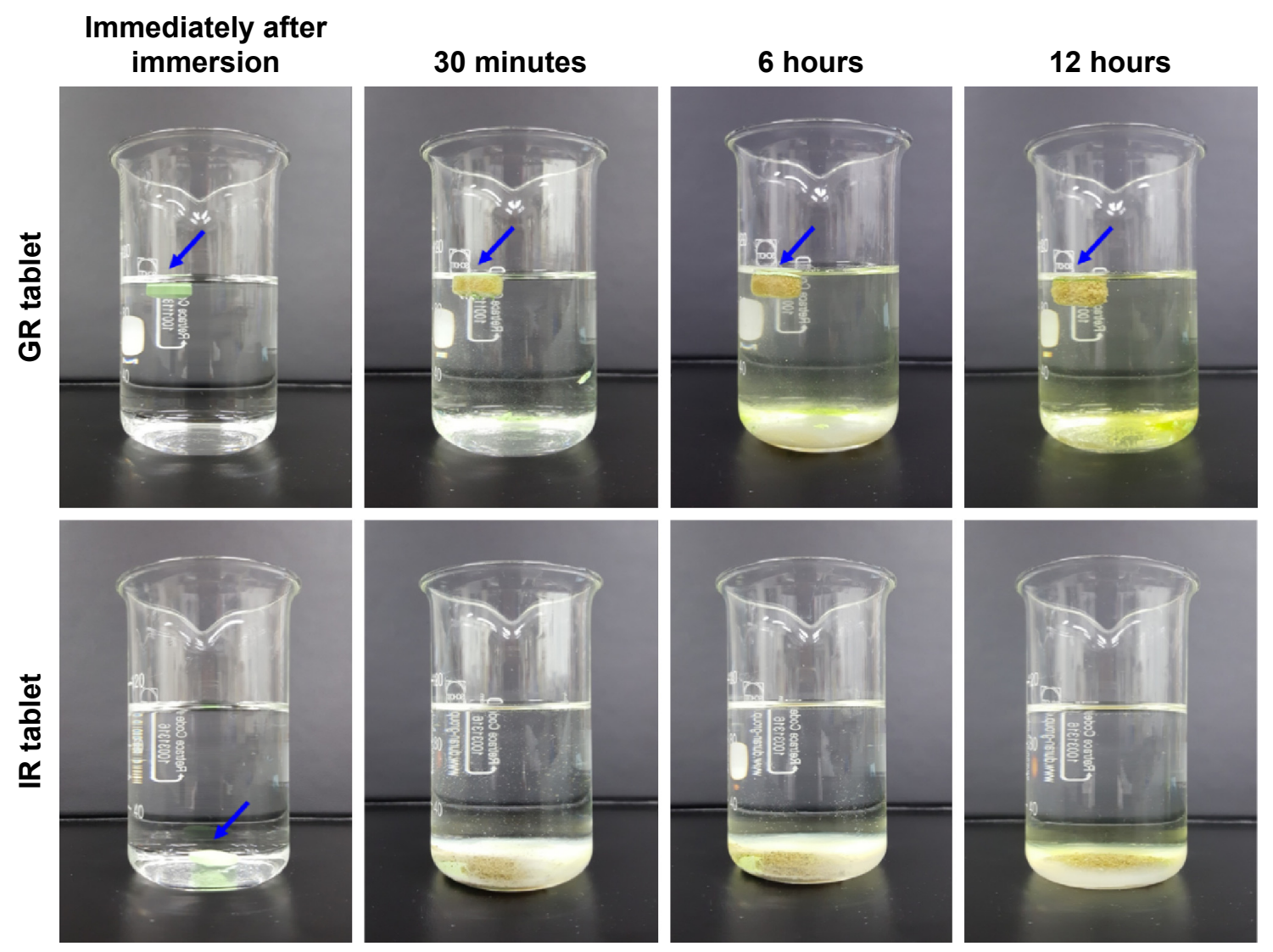

Figure I In vitro buoyancy behavior of GR tablet of DA-960 I as a function of time.

Notes: Arrows indicate the matrix maintaining its shape and integrity.

Abbreviations: DA-960I, a standardized ethanol extract of Artemisia asiatica; GR, gastroretentive; IR, immediate release.

gradually increased along with gradual erosion of the matrix for 8 hours. The accumulated amount of eupatilin released was quite linear and increased to $27 \%, 52 \%$, and $91 \%$ after 2, 4 and 8 hours, respectively. Table 2 represents the values for the correlation coefficient $\left(r^{2}\right)$, kinetic rate constant $(k)$, and release exponent $(n)$ after fitting the drug release

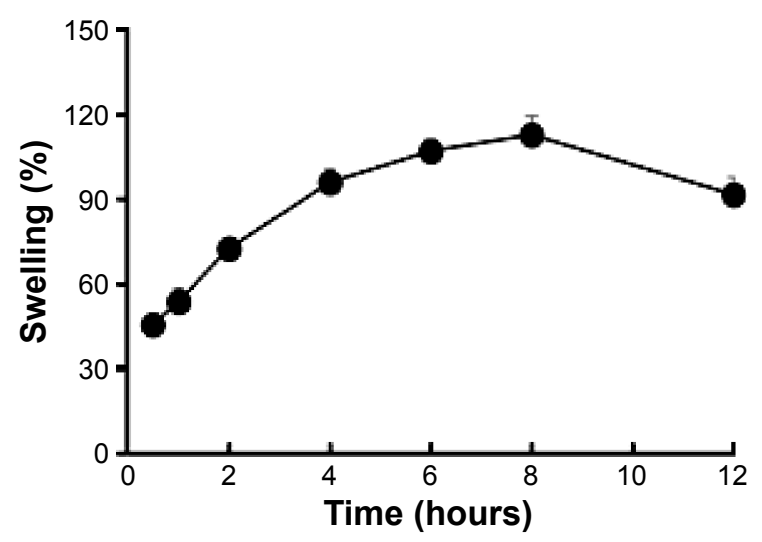

Figure 2 Swelling profile of the GR-tablet of DA-960I in acidic medium. Notes: Each value represents the mean \pm standard deviation $(n=6)$.

Abbreviations: DA-960I, a standardized ethanol extract of Artemisia asiatica; GR, gastroretentive. from the IR and GR tablets into the zero-order, first-order, Higuchi, and Korsmeyer-Peppas models. In vitro drug release profile from GR tablet was best fitted to zero-order and Korsmeyer-Peppas equations, showing high $r^{2}$ values of 0.997 and 0.996 , respectively. The " $n$ " value obtained from Korsmeyer-Peppas equation was $\sim 1.01$.

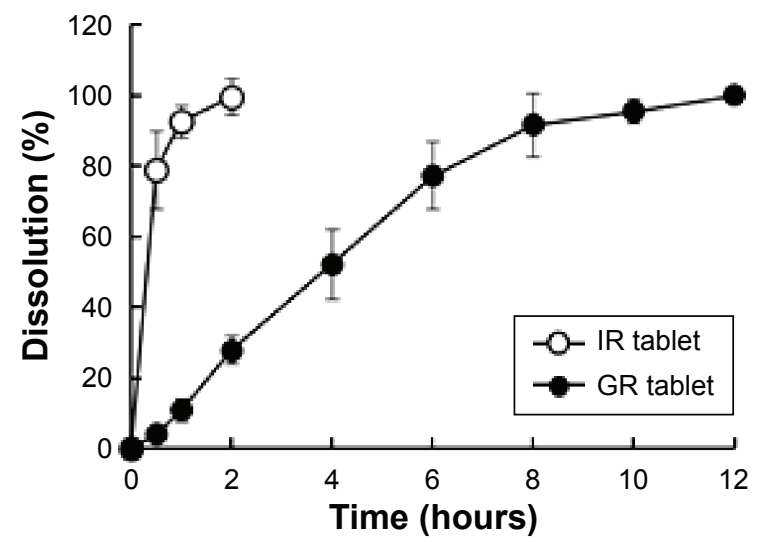

Figure 3 Dissolution profiles of eupatilin from IR and GR formulations in gastric juice.

Notes: The experiment was conducted in paddle method at $50 \mathrm{rpm}$ at $37^{\circ} \mathrm{C}$. Each value represents the mean \pm standard deviation $(n=6)$.

Abbreviations: GR, gastroretentive; IR, immediate release. 
Table 2 Kinetic parameters of eupatilin release from GR tablets

\begin{tabular}{llll}
\hline Equations & $\boldsymbol{k}$ & $\boldsymbol{r}^{2}$ & $\boldsymbol{n}$ \\
\hline Zero order & 13.25 & 0.997 & - \\
First order & 0.253 & 0.976 & - \\
Higuchi & 42.17 & 0.988 & - \\
Korsmeyer-Peppas & 0.127 & 0.996 & 1.01 \\
\hline
\end{tabular}

Abbreviations: GR, gastroretentive; $k$, slope; $n$, release exponent; $r^{2}$, coefficient of determination.

\section{In vivo radiographic studies}

The floating capacity of the barium sulfate-loaded GR tablet slightly decreased due to the introduction of a high-density radio opaque agent $\left(4.48 \mathrm{~g} / \mathrm{cm}^{3}\right)$. The floating duration of GR tablet decreased from 14 to 10 hours. Nevertheless, the barium sulfate-containing GR tablet met our requirements based on the criteria of the optimal floating lag time of $<3$ minutes and duration time of $>8$ hours (data not shown).

The representative photomicrographs are shown in Figure 4 and the position of tablet in the stomach is indicated by yellow open circles in the images. In the case of IR dosage form, the IR tablet was not found in the X-ray images after 30 minutes of oral administration. On the other hand, an in vivo X-ray imaging study clearly indicated that the prepared floating tablets of DA-9601 were retained over 4 hours in the upper part of small intestine of the beagle dogs. After 6 hours of administration, the floating tablet was not observed in the X-ray image.

\section{In vivo gastroprotective effect in gastritis- induced beagle dogs}

Beagle dogs with gastritis-like lesions manifested severe hemorrhage, erythema, and edema, with no significant differences in the severity levels between the animals. As shown in Figure 5, gastric endoscopies of beagle dogs in the control group administered placebo tablet showed a tendency toward a chronic condition and an incomplete recovery even after 15 days. On the other hand, oral administration of DA-9601 considerably reduced these symptoms in both IR and GR tablet-administered groups. Evaluation of the healing grade for the gastric wall (Figure 6) revealed that DA-9601 considerably reduced gastric mucosal lesions, with the healing starting 9 days after drug administration (healing grade $<3$ ) in both IR and GR tablet-treated groups. Accordingly, both DA-9601-treated groups resulted in a considerable reduction in values, showing significant differences from the placebo-treated group at 9, 12, and 15 days after administration. The healing grade in the GR tablet-treated group was equivalent to that of IR tablet-treated group, indicating that GR tablet was as effective as IR formulation, with reduced dosing frequency.

\section{Discussion}

DA-9601 is reported to possess strong antioxidative and cytoprotective actions in experimental animal models of mucosal damage, such as esophageal mucosal damage, ${ }^{21,22}$ and ethanolinduced gastritis. ${ }^{1}$ It was reported to block tumor necrosis factor alpha-induced interleukin- 8 production by mediating the inhibition of promoter activity as well as the nuclear factor- $\kappa \mathrm{B}$-dependent signaling system in inflammatory lesion. ${ }^{23}$ DA-9601 elevated the level of prostaglandin E2, protecting gastric mucosa from nonsteroidal anti-inflammatory drug-induced gastric damage. ${ }^{24}$ In this regard, we assumed that the GR system could be advantageous to amplify the
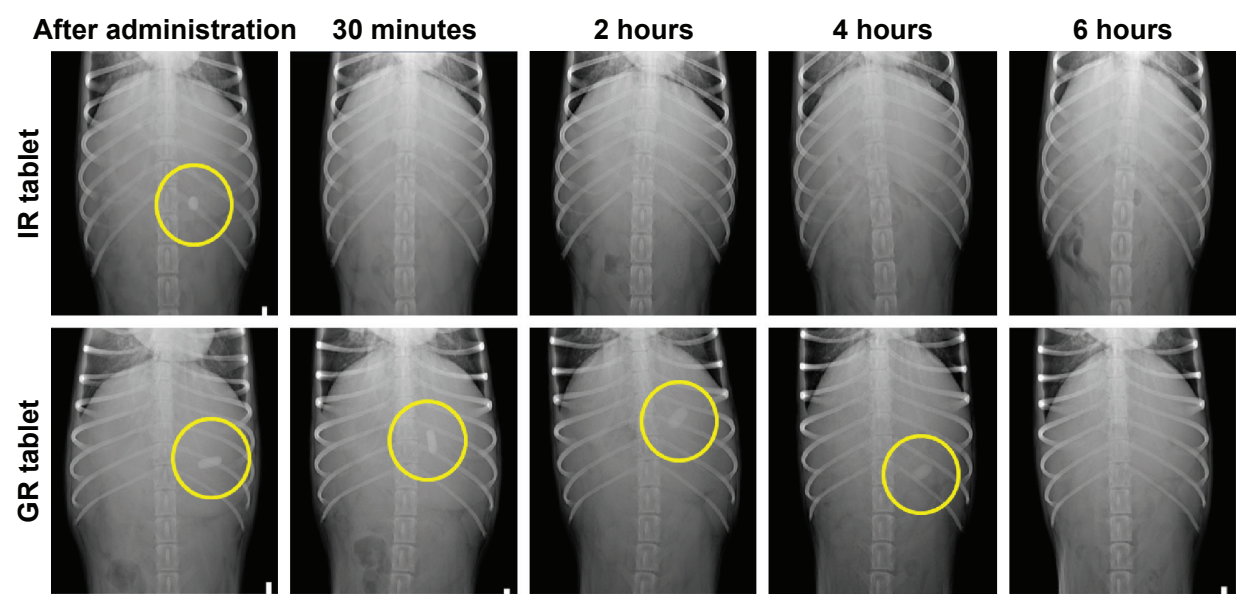

Figure 4 Representative radiographic images of the IR and GR tablets (yellow circles) of DA-960I after oral administration in beagle dogs.

Notes: Radiographs were taken using X-ray equipment after time intervals of immediately after oral administration and at $0.5,2,4$, and 6 hours after oral administration. Abbreviations: DA-960I, a standardized ethanol extract of Artemisia asiatica; GR, gastroretentive; IR, immediate release. 


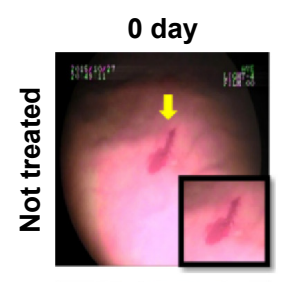

3 days
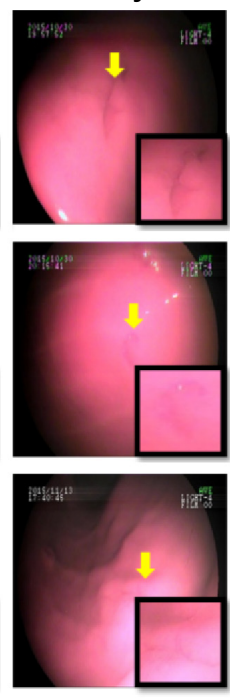

6 days
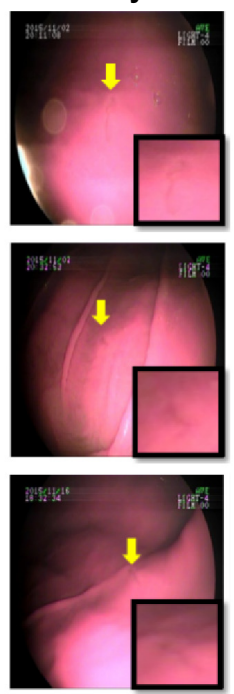

9 days
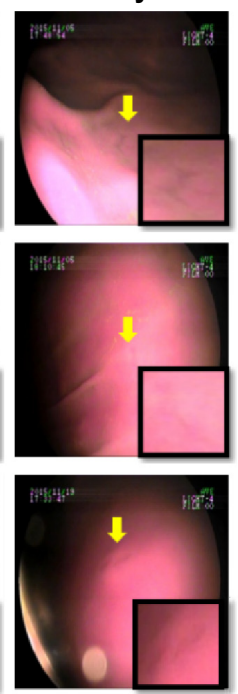

12 days
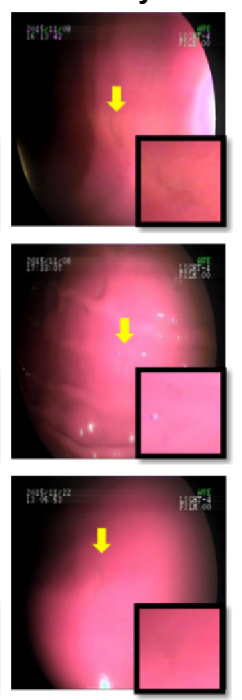

15 days
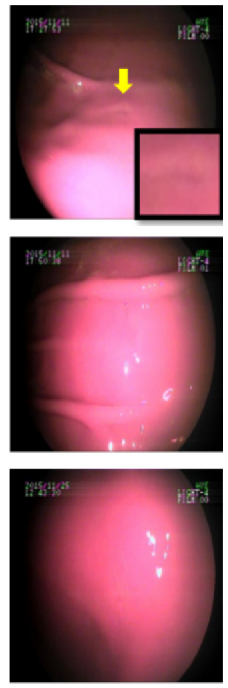

Figure 5 Endoscopic observation of gastritis-like lesions in the stomach of beagle dogs after oral administration of blank tablet (placebo), IR tablet, and GR tablet of DA-9601.

Notes: Images in the boxes are magnified $\times 2$ the original images. The arrow indicates gastritis-like lesions in the stomach.

Abbreviations: DA-960I, a standardized ethanol extract of Artemisia asiatica; GR, gastroretentive; IR, immediate release.

local gastroprotective activity of DA-9601, while reducing the dosing frequency. The GR tablet is principally composed of swellable hydrophilic polymers, low-density porous materials, sodium bicarbonate for carbon dioxide generation, an erosion polymer, and a surfactant to increase eupatilin solubility. ${ }^{12}$

Floating dosage forms should have a bulk density lower than that of gastric fluids to remain buoyant in the stomach

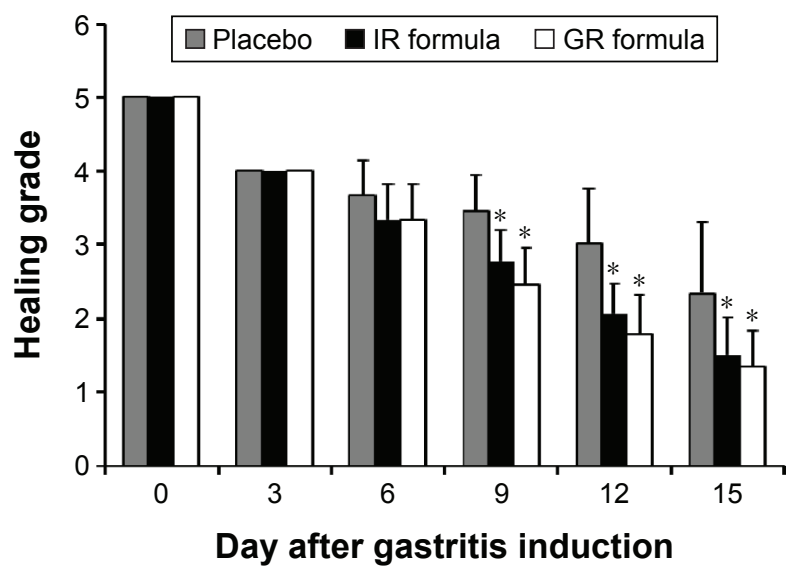

Figure 6 Gastroprotective effects of IR and GR tablets of DA-960I in experimentally induced gastritis in beagle dogs.

Notes: Grades indicate states of healing: grade 0, a normal gastric wall; grade I, presence of a scar with similar to normal gastric wall; grade 2, presence of an insignificant erosion with flare; grade 3, presence of severe erosion with flare and swelling; grade 4, presence of gastric ulcer with a blood spot; grade 5, presence of a gastric ulcer with bleeding. Asterisk (*) indicates statistically significant difference with placebo group $(P<0.05)$.

Abbreviations: DA-960I, a standardized ethanol extract of Artemisia asiatica; $\mathrm{GR}$, gastroretentive; IR, immediate release. with a prolonged retention time. A previous report stated that the floating system is strongly recommended to have an intrinsic density lower than stomach fluid $\left(1.004 \mathrm{~g} / \mathrm{cm}^{3}\right)$ to provide rapid onset of floating and prolonged floating duration in the stomach. ${ }^{25}$ Therefore, the effervescent GR tablet of DA-9601 was constructed to have a lower apparent density $\left(\sim 0.93 \mathrm{~g} / \mathrm{cm}^{3}\right)$. Actually, in our preliminary study, the lag time of floating tablet of DA-9601 showed a strong linear correlation with intrinsic density; as the intrinsic density of the GR tablet was decreased from 1.10 to $0.93 \mathrm{~g} / \mathrm{cm}^{3}$, the floating lag time was found to be drastically shortened from 9.4 to 0 (data not shown). It corresponds to the earlier report that GR matrices with an apparent density of $0.98 \mathrm{~g} / \mathrm{cm}^{3}$ had no floating lag time and floated for over 24 hours, while high-density tablet (density over $1.003 \mathrm{~g} / \mathrm{cm}^{3}$ ) did not float on the gastric fluid. ${ }^{11}$

Drug release from swollen and erodible matrices generally undertakes simultaneous matrix swelling, drug dissolution and diffusion into the aqueous medium, and polymer erosion and drug liberation. ${ }^{26,27}$ The release of eupatilin from the floating GR tablet was quite linear for 8 hours, showing over $90 \%$ of accumulated release rate. This dissolution profile suggests that the drug release rate was effectively controlled without dependence on the drug concentration in the matrix. In order to describe release mechanism from the GR tablet, the release exponent $(n)$ was calculated from KorsmeyerPeppas equation. The value of $n$ is utilized to characterize the release profile as either Fickian diffusion $n<0.5$, anomalous diffusion (non-Fickian) $(0.5<n<1)$, or zero-order release 
$(n=1) .{ }^{28}$ The $n$ value for DA-9601 GR tablet was 1.01, indicating that the drug release was predominantly controlled by the gradual erosion of the tablet dosage form.

In vivo radiographic studies provided straight evidence that the low-density GR tablet rapidly floated on the gastric fluid and maintained the buoyance condition over 4 hours in the upper part of small intestine (Figure 4), whereas the IR tablet was rapidly disintegrated and ingested in the stomach within 30 minutes. The location of the swollen tablet in the stomach was found to be continuously altered as a function of time, indicating that the GR tablets did not adhere to the gastric mucus, but floated on the gastric juice. On the other hand, in spite of the in vitro floating duration in acidic medium being over 12 hours, the in vivo residence time of the GR tablet measured by radiographic imaging was $<6$ hours. This difference between the in vitro and in vivo floating duration might be attributed to the addition of radio opaque agent for radiographic observation and/or the migrating myoelectric complex waves in the gastrointestinal tract. The addition of considerable quantity of barium sulfate ( $80 \mathrm{mg}$, density of $4.48 \mathrm{~g} / \mathrm{cm}^{3}$ ) to the GR formulation for $\mathrm{X}$-ray visualization led to the increment in apparent density and, thus, shortened the floating duration of the GR tablet. Moreover, the migrating motor complexes in the stomach perhaps considerably accelerated migration of the GR tablet from the stomach through the small intestine. It was reported that migration of myoelectric complex occurred approximately every 2 hours in healthy subjects and every hour in beagle dogs ${ }^{29}$ Actually, Baumgartner et $a{ }^{18}$ reported that the mean gastric residence time of HPMC-based floating tablet was $\sim 4$ hours in beagle dogs, despite the in vitro floating duration being over 8 hours.

In an in vivo efficacy study in gastric mucosal injured beagle dogs, gradual improvement of lesions was observed not only in DA-9601-treated groups but also in the placebotreated group (Figures 5 and 6 ) because gastric mucosal layer has several restoration mechanisms that protect and maintain epithelial integrity against physical disruption, such as villus contraction, migration of epithelial cells to cover the injured mucus, and closure of leaky epithelial intercellular spaces. ${ }^{30}$ It was reported that the restoration process was initiated within minutes after physical disruption and surface mucus cells were repaired within 3 days, whereas deeper mucosal cells was restored in 7 days. ${ }^{31}$ These restoration processes were significantly accelerated by DA-9601, showing significant differences from the placebo-treated group at 9, 12, and 15 days after lesion induction. Similarly, our previous study revealed that oral administration of DA-9601 at a dose of $40 \mathrm{mg} / \mathrm{kg}$ significantly reduced ethanol-induced gastric mucosal hemorrhagic lesions in rats. ${ }^{1}$ The authors suggested that the prevention and/or restoration effect of DA-9601 on gastric lesions was deeply associated with its inhibitory effect on xanthine oxidase activity and type conversion. ${ }^{1}$

\section{Conclusion}

In the present study, gastric residence time, dissolution profile, and pharmacological activity of twice-daily SR tablet of DA-9601 (Stillen 2X) were comprehensively evaluated. The GR dosage form floated onto gastric fluid with no lag time and kept the buoyancy for over 12 hours. The formula exhibited a linear release profile, achieving $>90 \%$ drug release for up to 12 hours. In vivo X-ray imaging study in beagle demonstrated that the GR tablet was located in the upper part of small intestine and remained there for over 4 hours. In addition, experimentally induced gastritis regions in the beagles treated with DA-9601 GR tablet healed quickly than those in the control group, and GR was as effective as IR tablet, with reduced dosing frequency. Therefore, we expect that the SR tablet of DA-9601 likely provides pharmacokinetic and/or pharmacological advantages over IR dosage forms, including maintaining constant therapeutic levels in the stomach for a longer period of time and improving patient compliance.

\section{Disclosure}

The authors report no conflicts of interest in this work.

\section{References}

1. Huh K, Kwon TH, Shin US, et al. Inhibitory effects of DA-9601 on ethanol-induced gastrohemorrhagic lesions and gastric xanthine oxidase activity in rats. J Ethnopharmacol. 2003;88:269-273.

2. Choi SM, Shim JH, Kang KK, Ahn BO, Yoo M. Gastroprotective effects of DA-6034, a new flavonoid derivative, in various gastric mucosal damage models. Dig Dis Sci. 2007;52:3075-3080.

3. Rouge N, Buio P, Doelker E. Drug absorption sites in the gastrointestinal tract and dosage forms for site-specific delivery. Int J Pharm. 1996;136: $117-139$.

4. Deshpande AA, Rhodes CT, Shah NH, Malick AW. Controlled-release drug delivery systems for prolonged gastric residence: an overview. Drug Dev Ind Pharm. 1996;10:539-631.

5. Davis SS. Formulation strategies for absorption windows. Drug Discov Today. 2005;10:249-257.

6. Priyadarshini R, Nandi G, Changder A, Chowdhury S, Chakraborty S, Ghosh LK. Gastroretentive extended release of metformin from methacrylamide-g-gellan and tamarind seed gum composite matrix. Carbohydr Polym. 2016;137:100-110.

7. Prajapati VD, Jani GK, Khutliwala TA, Zala BS. Raft forming system-an upcoming approach of gastroretentive drug delivery system. J Control Release. 2013;168:151-165.

8. Mostafavi A, Emami J, Varshosaz J, Davies NM, Rezazadeh M. Development of a prolonged-release gastroretentive tablet formulation of ciprofloxacin hydrochloride: pharmacokinetic characterization in healthy human volunteers. Int J Pharm. 2011;409:128-136. 
9. Brahma N, Kwon HK. Floating drug delivery systems: an approach to oral controlled drug delivery via gastric retention. J Controlled Release. 2000;63:235-259.

10. Streubel A, Siepmann J, Bodmeier R. Floating matrix tablets based on low density foam powder: effects of formulation and processing parameters on drug release. Eur J Pharm Sci. 2003;18:37-45.

11. Oh TO, Kim JY, Ha JM, et al. Preparation of highly porous gastroretentive metformin tablets using a sublimation method. Eur J Pharm Biopharm. 2013;83:460-467.

12. Jang SW. Development of Stillen GR with the application of gastroretentive floating technology. International Conference of the Korean Society of Pharmaceutical Sciences and Technology. Gyeonggi-do, Korea; 2015.

13. Rosa M, Zia H, Rhodes T. Dosing and testing in vitro of a bioadhesive and floating drug delivery system for oral application. Int J Pharm. 1994; 105:65-70.

14. Najib N, Suleiman MS. The kinetics of drug release from ethylcellulose solid dispersions. Drug Dev Ind Pharm. 1985;11:2169-2181.

15. Desai SJ, Singh P, Simonelli AP, Higuchi WI. Investigation of factors influencing release of solid drug dispersed in inert matrices III. Quantitative studies involving the polyethylene plastic matrix. J Pharm Sci. 1966;55:1230-1234.

16. Higuchi T. Mechanism of sustained-action medication. Theoretical analysis of rate of release of solid drugs dispersed in solid matrices. J Pharm Sci. 1963;52:1145-1149.

17. Korsmeyer RW, Gurny R, Doelker E, Buri P, Peppas NA. Mechanisms of solute release from porous hydrophilic polymers. Int J Pharm. 1983; 15:25-35.

18. Baumgartner S, Kristl J, Vrecer F, Vodopivec P, Zorko B. Optimisation of floating matrix tablets and evaluation of their gastric residence time. Int J Pharm. 2000;195:125-135.

19. Jang SW, Lee JW, Park SH, et al. Gastroretentive drug delivery system of DA-6034, a new flavonoid derivative, for the treatment of gastritis. Int J Pharm. 2008;356:88-94.

20. Goodman L, Torres B, Punke J, et al. Effects of firocoxib and tepoxalin on healing in a canine gastric mucosal injury model. $J$ Vet Intern Med. 2009;23:56-62.
21. Oh TY, Lee JS, Ahn BO, et al. Oxidative damages are critical in pathogenesis of reflux esophagitis: implication of antioxidants in its treatment. Free Radic Biol Med. 2001;30:905-915.

22. Lee JS, Oh TY, Ahn BO, et al. Involvement of oxidative stress in experimentally induced reflux esophagitis and Barrett's esophagus: clue for the chemoprevention of esophageal carcinoma by antioxidants. Mutat Res. 2001;480:189-200.

23. Choi SC, Choi EJ, Oh HM, et al. DA-9601, a standardized extract of Artemisia asiatica, blocks TNF-alpha-induced IL-8 and CCL20 production by inhibiting p38 kinase and NF-kappaB pathways in human gastric epithelial cells. World J Gastroenterol. 2006;12:4850-4858.

24. Oh TY, Ahn GJ, Choi SM, Ahn BO, Kim WB. Increased susceptibility of ethanol-treated gastric mucosa to naproxen and its inhibition by DA-9601, an Artemisia asiatica extract. World J Gastroenterol. 2005; 11:7450-7456.

25. Awasthi R, Kulkarni GT. Development and characterization of amoxicillin loaded floating microballoons for the treatment of Helicobacter pylori induced gastric ulcer. 2013;8:174-180.

26. Velasco MV, Ford JL, Rowe P, Rajabi-Siahboomi AR. Influence of drug: hydroxypropylmethylcellulose ratio, drug and polymer particle size and compression force on the release of diclofenac sodium from HPMC tablets. J Controlled Release. 1999;57:75-85.

27. Vázquez MJ, Pérez-Marcos B, Gómez-Amoza JL, Pacheco RI, Souto C, Concheiro A. Influence of technological variables on release of drugs from hydrophilic matrices. Drug Dev Ind Pharm. 1992;18: 1355-1375.

28. Peppas NA. Analysis of Fickian and non-Fickian drug release from polymers. Pharm Acta Helv. 1985;60:110-111.

29. Cunningham JG. Movements of the gastrointestinal tract. In: Cunningham JG, editor. Textbook of Veterinary Physiology. 2nd ed. Philadelphia: Saunders; 1997:272-289.

30. Blikslager AT, Moeser AJ, Gookin JL, Jones SL, Odle J. Restoration of barrier function in injured intestinal mucosa. Physiol Rev. 2007;87: $545-564$.

31. Strombeck DR, Guilford WG. Gastric structure and function. In: Strombeck DR, Guilford WG, editors. Small Animal Gastroenterology. 2nd ed. Davis CA: Stonegate Publishing Company; 1990:167-186.

\section{Publish your work in this journal}

Drug Design, Development and Therapy is an international, peerreviewed open-access journal that spans the spectrum of drug design and development through to clinical applications. Clinical outcomes, patient safety, and programs for the development and effective, safe, and sustained use of medicines are a feature of the journal, which

\section{Dovepress}

has also been accepted for indexing on PubMed Central. The manuscript management system is completely online and includes a very quick and fair peer-review system, which is all easy to use. Visit http://www.dovepress.com/testimonials.php to read real quotes from published authors. 